\title{
Pacific
}

Journal of

Mathematics

\section{A HYBRID OF THEOREMS OF VINOGRADOV AND PIATETSKI-SHAPIRO}

ANTAL BALOG AND JOHN BENJAMIN FRIEDLANDER 


\title{
A HYBRID OF THEOREMS OF VINOGRADOV AND PIATETSKI-SHAPIRO
}

\author{
ANTAL BALOG AND JohN Friedlander
}

\begin{abstract}
It was proved by Vinogradov that every sufficiently large odd integer can be written as the sum of three primes. We show that this remains the case when the primes so utilized are restricted to an explicit thin set. One may take, for example, the "Piatetski-Shapiro primes" $p=\left[n^{1 / \gamma}\right]$ with any $\gamma>20 / 21$. By a similar argument it would follow that, for arbitrary $\theta, 0<\theta<1$, and suitable $\lambda=\lambda(\theta)>0$, one may take the set of primes for which $\left\{p^{\theta}\right\}<p^{-\lambda}$.
\end{abstract}

1. Introduction. The ternary Goldbach problem was solved by Vinogradov [Vi] who gave an asymptotic formula for the number of representations of the (sufficiently large) odd integer $N$ as the sum of three primes. We state this in the form, cf. [Va],

$$
\begin{aligned}
R(N) & \stackrel{\text { def }}{=} \sum_{p_{1}+p_{2}+p_{3}=N}\left(\log p_{1}\right)\left(\log p_{2}\right)\left(\log p_{3}\right) \\
& =\frac{1}{2} \mathfrak{S}(N) N^{2}+O_{A}\left(\frac{N^{2}}{\log ^{A} N}\right)
\end{aligned}
$$

valid for arbitrary $A>0$, where $\mathfrak{S}(N)$ is the singular series

$$
\mathfrak{S}(N)=\prod_{p \mid N}\left(1-\frac{1}{(p-1)^{2}}\right) \prod_{p \nmid N}\left(1+\frac{1}{(p-1)^{3}}\right) .
$$

Wirsing [Wi], motivated by earlier work of Erdős and Nathanson [EN] on sums of squares, considered the question of whether one could find thin subsets $S$ of primes which were still sufficient to obtain all sufficiently large odd integers as sums of three of them. He obtained the very satisfactory answer that there exist such sets $S$ with the property that $\sum_{p \leq x, p \in S} 1 \ll(x \log x)^{1 / 3}$. This result was later rediscovered by Ruzsa. Wirsing's result, which is obviously best possible apart from the logarithmic factor, is based on probabilistic considerations and does not lead to a subset of the primes which is constructive or recognizable.

It was Wolke who suggested the problem of finding more familiar thin sets of primes which serve this purpose and he announced his results on one such construction at an Oberwolfach meeting in 1986. 
There are not many thin sets of primes about which we have very much information. One may expect for example that we should first be able to obtain an asymptotic estimate for the counting function of such a set and this already bars many sets from consideration. An exception is provided by a theorem of Piatetski-Shapiro.

We fix a real number $c$ and consider the number of $n \leq x$ such that the integer part $\left[n^{c}\right]$ is a prime. In the case that $0<c \leq 1$ every prime $\leq x^{c}$ occurs in this fashion and it is a simple consequence of the prime number theorem that we have the expected asymptotic formula

$$
\sum_{\substack{n \leq x \\\left[n^{c}\right]=p}} 1=(1+o(1)) \frac{x}{c \log x}
$$

Piatetski-Shapiro [PS] proved the much more difficult result that the asymptotic formula (1.3) still holds in the range $1<c<12 / 11$. This range for $c$ has been improved since by a number of authors; more recently Heath-Brown [HB] has extended it to $1<c<1.14 \ldots$ and Kolesnik [Ko] further to $1<c<39 / 34$.

We let $\gamma=1 / c$, so that in the interesting case we have $0<\gamma<1$, and note that by $(1.3)$

$$
P_{\gamma}(x) \stackrel{\text { def }}{=} \sum_{\substack{p \leq x \\ p=\left[n^{1 / \gamma}\right]}} 1=(1+o(1)) \frac{x^{\gamma}}{\log x}
$$

so that this set $P_{\gamma}$ of "Piatetski-Shapiro primes of type $\gamma$ " form a thin set of primes. In this paper we show that, provided $\gamma$ is not too much smaller than 1 , this thin set $P_{\gamma}$ suffices for the ternary Goldbach problem.

Theorem 1. Let $\gamma_{1}, \gamma_{2}, \gamma_{3}$ be fixed subject to $0<\gamma_{i} \leq 1$ and

$$
9\left(1-\gamma_{3}\right)<1
$$

$$
9\left(1-\gamma_{2}\right)+6\left(1-\gamma_{3}\right)<1
$$

$$
9\left(1-\gamma_{1}\right)+6\left(1-\gamma_{2}\right)+6\left(1-\gamma_{3}\right)<1
$$


Then for any $A>0$ and with $\mathfrak{S}(N)$ defined by (1.2) we have

$$
\text { (1.7) } \begin{aligned}
T(N) & \stackrel{\text { def }}{=} \frac{1}{\gamma_{1} \gamma_{2} \gamma_{3}} \sum_{\substack{p_{1}+p_{2}+p_{3}=N \\
p_{i} \in P_{\gamma_{t}}}} p_{1}^{1-\gamma_{1}}\left(\log p_{1}\right) p_{2}^{1-\gamma_{2}}\left(\log p_{2}\right) p_{3}^{1-\gamma_{3}}\left(\log p_{3}\right) \\
& =\frac{1}{2} \mathfrak{S}(N) N^{2}+O_{A}\left(\frac{N^{2}}{\log ^{A} N}\right) .
\end{aligned}
$$

This means in particular that we may require the three summands to be Piatetski-Shapiro primes of different type. However, choosing $\gamma_{1}=\gamma_{2}=\gamma_{3}=\gamma$ we obtain

Corollary 1. For any fixed $20 / 21<\gamma \leq 1$ the primes $p$ of the form $\left[n^{1 / \gamma}\right]$ have the property that every sufficiently large odd integer can be written as the sum of three of them.

Also, choosing $\gamma_{1}=\gamma_{2}=1$ we obtain

COROllary 2. For any fixed $8 / 9<\gamma \leq 1$ every sufficiently large odd integer can be written as the sum of three primes one of which is of the form $\left[n^{1 / \gamma}\right]$.

Note that $8 / 9<\gamma$ is not much worse than the best known results for the existence of Piatetski-Shapiro primes.

We find it somewhat more convenient to weight the primes in (1.7); the unweighted version is given by

THEOREM 2. With $\gamma_{1}, \gamma_{2}, \gamma_{3}$ and $\mathfrak{S}(N)$ as in Theorem 1, we have

$$
\sum_{\substack{p_{1}+p_{2}+p_{3}=N \\ p_{i} \in P_{\gamma_{t}}}} 1=(1+o(1)) \frac{\gamma_{1} \gamma_{2} \gamma_{3} \Gamma\left(\gamma_{1}\right) \Gamma\left(\gamma_{2}\right) \Gamma\left(\gamma_{3}\right)}{\Gamma\left(\gamma_{1}+\gamma_{2}+\gamma_{3}\right)} \frac{\mathfrak{S}(N) N^{\gamma_{1}+\gamma_{2}+\gamma_{3}-1}}{\log ^{3} N} \text {. }
$$

The condition that $p$ be a Piatetski-Shapiro prime of type $\gamma$ is roughly speaking equivalent to the fact that the fractional part $\left\{p^{\gamma}\right\}$ is $\left\langle p^{-\lambda}\right.$ with $\lambda=1-\gamma$. The method we give here works also for the more general situation and with tiny modifications the proof of Theorem 1 gives in particular

Theorem 3. For any fixed $22 / 25<\gamma \leq 1$ the set of primes $p$ satisfying $\left\{p^{\gamma}\right\}<p^{-2(1-\gamma) / 3}$ has the property that every sufficiently large odd integer is the sum of three of them. 
We note that the number of primes $\leq x$ in the above set is $\ll$ $x^{(1+2 \gamma) / 3}$ making it a thinner set (in the best case, density $\left.x^{23 / 25+\varepsilon}\right)$ than that given by Corollary 1 . The choice $\lambda=2(1-\gamma) / 3$ seems to be the optimal one in this regard from the point of view of our method.

For the proof of Theorem 1 we do not directly use the HardyLittlewood method. The fact that we are dealing with a thin set of primes would require us to save a fixed power in the minor arc estimates and this would necessitate a choice of the major arcs so numerous as to require information about the distribution of primes in arithmetic progressions which is currently unavailable. Instead we are able to reduce the problem of estimating (1.7) to the Vinogradov result (1.1). In the same fashion Theorem 2, whose proof we do not give, can be reduced to a weighted version of (1.1) which seems not to have appeared in the literature but which can be proved by the same classical method [Va] that gives (1.1).

We may remark that the error term in (1.7) comes not from the reduction to (1.1) but rather from the proof of (1.1) itself; in fact we prove that for a suitable $0<\varepsilon=\varepsilon(\gamma)$, we have $T(N)=R(N)+$ $O\left(N^{2-\varepsilon}\right)$.

2. Preliminaries. The reduction of Theorem 1 to the Vinogradov estimate (1.1) is by means of the identity

$$
\begin{aligned}
f_{1} f_{2} f_{3}-g_{1} g_{2} g_{3}= & \left(f_{1}-g_{1}\right) f_{2} f_{3} \\
& +g_{1}\left(f_{2}-g_{2}\right) f_{3}+g_{1} g_{2}\left(f_{3}-g_{3}\right) .
\end{aligned}
$$

We let $g_{1}=g_{2}=g_{3}=g(\alpha)=\sum_{p<N} e(\alpha p) \log p$ so that the sum in (1.1) is given by

$$
R(N)=\int_{0}^{1} g^{3}(\alpha) e(-N \alpha) d \alpha
$$

We let, for $1 \leq i \leq 3$,

$$
f_{i}(\alpha)=\frac{1}{\gamma_{i}} \sum_{p<N} e(\alpha p) p^{1-\gamma_{i}} \log p\left(\left[-p^{\gamma_{i}}\right]-\left[-(p+1)^{\gamma_{i}}\right]\right)
$$

so that the sum in (1.7) is given by

$$
T(N)=\int_{0}^{1} f_{1}(\alpha) f_{2}(\alpha) f_{3}(\alpha) e(-N \alpha) d \alpha .
$$


Thus, by (2.1), we have $T(N)=R(N)+E$, where

$$
\begin{aligned}
\text { (2.2) } E \ll & \left(\sup _{\alpha}\left|f_{1}-g\right|\right) \int_{0}^{1}\left|f_{2} f_{3}\right| d \alpha+\left(\sup _{\alpha}\left|f_{2}-g\right|\right) \int_{0}^{1}\left|g f_{3}\right| d \alpha \\
& +\left(\sup _{\alpha}\left|f_{3}-g\right|\right) \int_{0}^{1}|g|^{2} d \alpha .
\end{aligned}
$$

We estimate the integrals in (2.2) by Cauchy's inequality and Parseval's identity. We appeal to a sieve bound of Deshouillers [De] by which, for any $\gamma$

$$
\sum_{\substack{p \leq x \\ p=\left[n^{1 / \gamma}\right]}} 1 \ll \frac{x^{\gamma}}{\log x}
$$

(Actually, for our range of $\gamma$, even the asymptotic formula is available, and the result follows also from Theorem 4 below.) Inserting these we find

$$
\begin{aligned}
\int_{0}^{1}|g|^{2} d \alpha & \ll N \log N, \\
\int_{0}^{1}\left|f_{3} g\right| d \alpha & \ll\left(\int_{0}^{1}\left|f_{3}\right|^{2} d \alpha\right)^{1 / 2}\left(\int_{0}^{1}|g|^{2} d \alpha\right)^{1 / 2} \\
& \ll N^{3 / 2-\gamma_{3} / 2} \log N, \\
\int_{0}^{1}\left|f_{2} f_{3}\right| d \alpha & \ll N^{2-\gamma_{2} / 2-\gamma_{3} / 2} \log N,
\end{aligned}
$$

so that we require, for $1 \leq i \leq 3$, an estimate

$$
\sup _{\alpha}\left|f_{i}-g\right| \ll N^{1-\delta_{i}-\varepsilon},
$$

for some $\varepsilon>0$, where $\delta_{3}=0, \delta_{2}=\frac{1}{2}\left(1-\gamma_{3}\right)$, and $\delta_{1}=\frac{1}{2}\left(1-\gamma_{2}\right)+$ $\frac{1}{2}\left(1-\gamma_{3}\right)$.

By (1.4)-(1.6) we see that Theorem 1 follows from

TheOREM 4. Let $\gamma, \delta$ satisfy $0<\gamma \leq 1,0<\delta$ and

$$
9(1-\gamma)+12 \delta<1 \text {. }
$$

Then, uniformly in $\alpha$, we have

$$
\frac{1}{\gamma} \sum_{\substack{p<N \\ p=\left[n^{1 / \gamma}\right]}} e(\alpha p) p^{1-\gamma} \log p=\sum_{p<N} e(\alpha p) \log p+O\left(N^{1-\delta}\right),
$$

where the implied constant may depend on $\gamma$ and $\delta$ only. 
From now on implied constants may depend on $\varepsilon, \gamma, \delta$ but not on $\alpha$. The special case $\alpha=0$ gives a Piatetski-Shapiro theorem and indeed the following arguments are, to a large extent, based on the proof of that theorem given in [HB].

Denoting the left-hand side of $(2.4)$ by $f(\alpha)$, we have

$$
f(\alpha)=\frac{1}{\gamma} \sum_{p<N} e(\alpha p) p^{1-\gamma} \log p\left(\left[-p^{\gamma}\right]-\left[-(p+1)^{\gamma}\right]\right)
$$

and

$$
\begin{aligned}
f(\alpha)-g(\alpha)= & \frac{1}{\gamma} \sum_{p<N} e(\alpha p) p^{1-\gamma} \log p\left(\psi\left(-(p+1)^{\gamma}\right)-\psi\left(-p^{\gamma}\right)\right) \\
& +O(\log N) \\
= & \frac{1}{\gamma} \sum_{k<N} \Lambda(k) e(\alpha k) k^{1-\gamma}\left(\psi\left(-(k+1)^{\gamma}\right)-\psi\left(-k^{\gamma}\right)\right) \\
& +O\left(N^{3 / 2-\gamma}\right)
\end{aligned}
$$

where $\psi(t)=\{t\}-\frac{1}{2}$. The error term is admissible since $2(1-\gamma)+$ $2 \delta<1$.

We introduce the notation $n \sim N$ to signify that $n$ runs through the integers $N<n \leq N^{\prime}$ for some $N^{\prime} \leq 2 N$. The actual choice of $N^{\prime}$ may change as we proceed. The same is ture of the positive constant $\varepsilon$. On the other hand, $n \asymp N$ means $N \ll n \ll N$.

Theorem 4 will follow if we prove for each $1 \leq x \leq N$ the estimate

$$
\sum_{k \sim x} \Lambda(k) e(\alpha k) k^{1-\gamma}\left(\psi\left(-(k+1)^{\gamma}\right)-\psi\left(-k^{\gamma}\right)\right) \ll x^{1-\delta} .
$$

We use the well-known expansions

$$
\begin{aligned}
\psi(t)= & -\sum_{0<|h| \leq H_{0}} \frac{1}{2 \pi i h} e(h t)+O\left(\min \left(1, \frac{1}{H_{0}\|t\|}\right)\right), \\
& \min \left(1, \frac{1}{H_{0}\|t\|}\right)=\sum_{h=-\infty}^{\infty} b_{h} e(h t),
\end{aligned}
$$

where $\|t\|=\min (\{t\}, 1-\{t\})$ and

$$
\left|b_{h}\right| \ll \min \left(\frac{\log 2 H_{0}}{H_{0}}, \frac{H_{0}}{h^{2}}\right) .
$$

We insert (2.6) into (2.5) and estimate first the contribution of the error term. For this purpose and also for later use we recall a familiar estimate of van der Corput (see, for instance, [Ti, Theorem 5.9]). 
LEMMA 1. If $\Delta>0$ and $F^{\prime \prime}(t) \asymp \Delta\left(\right.$ or $\left.-F^{\prime \prime}(t) \asymp \Delta\right)$ for $N<t \leq$ $2 N$ then

$$
\sum_{n \sim N} e(F(n)) \ll N \Delta^{1 / 2}+\Delta^{-1 / 2}
$$

Using (2.7) and trivial estimates we find that the contribution to (2.5) from the error term in (2.6) is bounded as

$$
\begin{aligned}
& \ll x^{1-\gamma+\varepsilon} \sum_{h=-\infty}^{\infty}\left|b_{h}\right|\left|\sum_{k \sim x} e\left(h k^{\gamma}\right)\right| \\
& \ll H_{0}^{-1} x^{2-\gamma+\varepsilon}+x^{1-\gamma+\varepsilon} \sum_{h=1}^{\infty}\left|b_{h}\right|\left(h^{1 / 2} x^{\gamma / 2}+h^{-1 / 2} x^{1-\gamma / 2}\right) \\
& \ll\left(H_{0}^{-1} x^{2-\gamma}+H_{0}^{1 / 2} x^{1-\gamma / 2}\right) x^{\varepsilon} .
\end{aligned}
$$

We fix the choice $H_{0}=x^{1-\gamma+\delta+\varepsilon}$ and find that this contribution is $\ll x^{1-\delta}$ as required since $2(1-\gamma)+3 \delta<1$. As for the sum over $h$ occurring in (2.6) we may partition it into $\ll \log x$ subsums of the type $h \sim H$ for various $H \leq H_{0}$. (We shall ignore negative $H$ which may be treated in the same fashion; in fact they give the same contribution in absolute value as do the positive $H$ for $-\alpha$.) The proof of (2.5) thus reduces to showing that, for each such $H$, we have

$$
\sum_{h \sim H} \frac{1}{h}\left|\sum_{k \sim x} \Lambda(k) k^{1-\gamma} e(\alpha k)\left(e\left(h(k+1)^{\gamma}\right)-e\left(h k^{\gamma}\right)\right)\right| \ll x^{1-\delta-\varepsilon} .
$$

When $H \leq H_{1} \stackrel{\text { def }}{=} x^{1-\gamma}$ we write

$$
e\left(h(k+1)^{\gamma}\right)-e\left(h k^{\gamma}\right)=2 \pi i \gamma h \int_{0}^{1}(k+u)^{\gamma-1} e\left(h(k+u)^{\gamma}\right) d u .
$$

When $H>H_{1}$ we treat these two terms separately. After partial summation, we reduce the proof of (2.9) to the problem of showing that for each $1 \leq x \leq N, 0 \leq u \leq 1, H \leq H_{0}$,

$$
\min \left(1, \frac{H_{1}}{H}\right) \sum_{h \sim H}\left|\sum_{k \sim x} \Lambda(k) e\left(\alpha k+h(k+u)^{\gamma}\right)\right| \ll x^{1-\delta-\varepsilon} .
$$

3. Some combinatorics. A special case of the identity of HeathBrown [HB2] is given by

$$
-\frac{\zeta^{\prime}}{\zeta}=-\frac{\zeta^{\prime}}{\zeta}(1-Z \zeta)^{3}-\sum_{j=1}^{3}\left(\begin{array}{l}
3 \\
j
\end{array}\right)(-1)^{j} Z^{j} \zeta^{j-1}\left(-\zeta^{\prime}\right)
$$


where $Z=Z(s)=\sum_{m \leq x^{1 / 3}} \mu(m) m^{-s}$. From this we can decompose $\Lambda(k)$ for $k \sim x$ as

$$
\Lambda(k)=\sum_{j=1}^{3}\left(\begin{array}{l}
3 \\
j
\end{array}\right)(-1)^{j-1} \sum_{m_{1} \cdots m_{2 j}=k} \mu\left(m_{1}\right) \cdots \mu\left(m_{j}\right) \log m_{2 j} .
$$

For any arithmetic function $G(k)$ we can express $\sum_{k \sim x} \Lambda(k) G(k)$ in terms of sums

$$
\sum_{\substack{m_{1} \cdots m_{2 j} \sim x \\ m_{\imath} \sim M_{\imath}}} \cdots\left(m_{1}\right) \cdots \mu\left(m_{j}\right) \log m_{2 j} G\left(m_{1} \cdots m_{2 j}\right)
$$

where $j \leq 3, M_{1} \cdots M_{2 j} \sim x$ and $M_{1}, \ldots, M_{j} \leq x^{1 / 3}$. By dividing the $M_{j}$ into two groups we have

$$
\left|\sum_{k \sim x} \Lambda(k) G(k)\right| \ll_{\varepsilon} x^{\varepsilon} \max \left|\sum_{\substack{m n \sim x \\ m \sim M}} \sum_{\substack{\sim \\ \sim}} a_{m} b_{n} G(m n)\right|
$$

where the maximum is taken over all bilinear forms with coefficients satisfying one of

$$
\left|a_{m}\right| \leq 1, \quad\left|b_{n}\right| \leq 1
$$

or

$$
\left|a_{m}\right| \leq 1, \quad b_{n}=1
$$

or

$$
\left|a_{m}\right| \leq 1, \quad b_{n}=\log n
$$

and also satisfying in all cases

$$
M \leq x
$$

We refer to the case (3.3) as being a Type II sum and to the other cases as being Type I sums.

By dividing the $M_{j}$ into two groups in a judicious fashion we are able to reduce the range of $M$ from (3.6).

Proposition 1. If we have real numbers $0<a<1,0<b<c<$ ? satisfying

$$
\begin{gathered}
b<\frac{2}{3}, \\
1-c<c-b,
\end{gathered}
$$


and

$$
1-a<\frac{1}{2} c
$$

then (3.2) still holds when (3.6) is replaced by the conditions

$$
M \leq x^{a} \text { for Type I sums, }
$$

$$
x^{b} \leq M \leq x^{c} \text { for Type II sums. }
$$

To prove this first notice that if there is an $M_{i}$ satisfying $x^{1-c}<$ $M_{i}<x^{1-b}$ then we are done by taking a Type II sum with $M$ equal to the product to the other factors. (The possibility that $\log m_{2 j}>1$ is unimportant, due to the presence of $x^{\varepsilon}$ in (3.2).) In case not, let $M_{0}$ be the product of those $M_{i} \leq x^{1-c}$. If $M_{0}>x^{1-c}$ then by (3.8) there is a subproduct between $x^{1-c}$ and $x^{1-b}$. Choosing $M$ to be the product of the other factors we get an admissible Type II sum. If, on the other hand, $M_{0} \leq x^{1-c}$ we use the fact that, because $1-b>\frac{1}{3}$ by (3.7) we have at most two additional $M_{i}$ not in $M_{0}$ and these occur with coefficient 1 or $\log n$. Then by (3.9) we have an admissible Type I sum where $M$ is the product of all $M_{i}$ but the largest. This yields Proposition 1.

For the application of Proposition 1 to our situation we write

$$
S_{\mathrm{II}}=\min \left(1, \frac{H_{1}}{H}\right) \sum_{h \sim H}\left|\sum_{\substack{m n \sim \mathcal{X} \\ m \sim M}} \sum_{m} a_{m} b_{n} e\left(\alpha m n+h(m n+u)^{\gamma}\right)\right|
$$

where the coefficients satisfy (3.3) and $M$ is in the range (3.11) while we write $S_{I}$ when the coefficients satisfy (3.4) or (3.5) and $M$ is in the range (3.10).

4. Type II bilinear forms. For any fixed $0 \leq u \leq 1, H \leq H_{0}$ we choose the coefficients $c_{h}, h \sim H$ such that

$$
\begin{aligned}
& \left|\sum_{\substack{m n \sim x \\
m \sim M}} a_{m} b_{n} e\left(\alpha m n+h(m n+u)^{\gamma}\right)\right| \\
& \quad=c_{h} \sum_{\substack{m n \sim x \\
m \sim M}} a_{m} b_{n} e\left(\alpha m n+h(m n+u)^{\gamma}\right) .
\end{aligned}
$$

Obviously $\left|c_{h}\right|=1$. Let $Q \geq 1$ be a parameter to be chosen later optimally and we preselect the pairs $(h, n)$ according to the size of 
$h n^{\gamma}$; precisely for $q \sim Q$

$$
\begin{aligned}
W_{q}=\{(h, n), h \sim H, & n \sim x / m, \\
& \left.H x^{\gamma}(q-1) / Q<h n^{\gamma} M^{\gamma} \leq H x^{\gamma} q / Q\right\} .
\end{aligned}
$$

From the Cauchy inequality we have

$$
\begin{aligned}
& \left(\sum_{h \sim H} \sum_{\substack{m n \sim \mathcal{X} \\
m \sim M}} a_{m} b_{n} c_{h} e\left(\alpha m n+h(m n+u)^{\gamma}\right)\right)^{2} \\
& \leq Q M \sum_{q \sim Q} \sum_{m \sim M}\left|\sum_{(h, n) \in W_{q}} b_{n} c_{h} e\left(\alpha m n+h(m n+u)^{\gamma}\right)\right|^{2} \\
& \leq Q M \sum_{q \sim Q} \sum_{\substack{\left(h_{1}, n_{1}\right) \in W_{q} \\
\left(h_{2}, n_{2}\right) \in W_{q}}} \mid \sum_{m} e\left(\alpha\left(n_{1}-n_{2}\right) m\right. \\
& \left.+h_{1}\left(m n_{1}+u\right)^{\gamma}-h_{2}\left(m n_{2}+u\right)^{\gamma}\right) \\
& \leq Q M \sum_{\substack{h_{1} \sim H, h_{2} \sim H, n_{1} \sim x / M, n_{2} \sim x / M \\
M^{\gamma}\left|h_{1} n_{1}^{\gamma}-h_{2} n_{2}^{\gamma}\right| \leq H x^{\gamma} / Q}} \mid \sum_{m} e\left(\alpha\left(n_{1}-n_{2}\right) m\right. \\
& \left.+h_{1}\left(m n_{1}+u\right)^{\gamma}-h_{2}\left(m n_{2}+u\right)^{\gamma}\right)
\end{aligned}
$$

where the innermost sum over $m$ is taken in an interval defined by $m \sim M, m \sim x / n_{1}, m \sim x / n_{2}$. We are going to apply Lemma 1 . Writing

$$
F(m)=\alpha\left(n_{1}-n_{2}\right) m+h_{1}\left(m n_{1}+u\right)^{\gamma}-h_{2}\left(m n_{2}+u\right)^{\gamma}
$$

we can quickly calculate that for $m \sim M$

$$
\begin{aligned}
F^{\prime \prime}(m) & =\gamma(\gamma-1) h_{1} n_{1}^{2}\left(m n_{1}+u\right)^{\gamma-2}-\gamma(\gamma-1) h_{2} n_{2}^{2}\left(m n_{2}+u\right)^{\gamma-2} \\
& =\gamma(\gamma-1) m^{\gamma-2}\left(h_{1} n_{1}^{\gamma}-h_{2} n_{2}^{\gamma}\right)+O\left(\frac{H}{H_{1} M^{2}}\right) \asymp \frac{V}{M^{2}}
\end{aligned}
$$

whenever $\frac{1}{2} V<M^{\gamma}\left|h_{1} n_{1}^{\gamma}-h_{2} n_{2}^{\gamma}\right| \leq V$ and $H / H_{1} \ll V \leq H x^{\gamma} / Q$.

Let $S(V)$ be the number of quadruples $\left(h_{1}, h_{2}, n_{1}, n_{2}\right)$ satisfying $h_{1} \sim H, h_{2} \sim H, n_{1} \sim x / M, n_{2} \sim x / M$ and $M^{\gamma}\left|h_{1} n_{1}^{\gamma}-h_{2} n_{2}^{\gamma}\right| \leq V$.

The sum over $m$ is bounded trivially by $M$ for quadruples corresponding either to $V \leq 1$ or to $V \ll H / H_{1}$, and we use (2.7) for the 
others. We distinguish the cases $H \leq H_{1}$ and $H_{1} \leq H \leq H_{0}$. We arrive at

(4.1) $S_{\mathrm{II}} \ll\left(Q M^{2} S(1)+Q M \sum_{V \leq H x^{\gamma} / Q} S(V)\left(V^{1 / 2}+\frac{M}{V^{1 / 2}}\right)\right)^{1 / 2}$ for $H \leq H_{1}$ and

(4.2) $S_{\text {II }} \ll \frac{H_{1}}{H}\left(Q M^{2} S\left(\frac{H}{H_{1}}\right)\right.$

$$
\left.+Q M \sum_{H / H_{1} \ll V \leq H x^{\gamma} / Q} S(V)\left(V^{1 / 2}+\frac{M}{V^{1 / 2}}\right)\right)^{1 / 2}
$$

for $H_{1} \leq H \leq H_{0}$. Note that in these sums $V$ runs through powers of 2. The necessary bound for $S(V)$ is contained in the next lemma due to Heath-Brown [HB].

Lemma 2. Let $H \geq 1, N \geq 1, V>0$ and $\gamma \neq 0$. We have

$$
\begin{aligned}
\#\left\{\left|\frac{h_{1}}{h_{2}}-\left(\frac{n_{1}}{n_{2}}\right)^{\gamma}\right|\right. & \left.\leq V: h_{1} \sim H, h_{2} \sim H, n_{1} \sim N, n_{2} \sim N\right\} \\
& \ll V H^{2} N^{2}+H N \log (2 H N) .
\end{aligned}
$$

This immediately gives that

$$
S(V) \ll \frac{V H x^{2-\gamma}}{M^{2}}+\frac{H x \log x}{M} .
$$

Inserting (4.3) into (4.1) and (4.2) we get that, in the case of $H \leq H_{1}$,

$$
\begin{aligned}
S_{\text {II }} \ll & M^{1 / 2} Q^{1 / 2} H^{1 / 2} x^{1 / 2+\varepsilon}+\frac{H^{5 / 4} x^{1+\gamma / 4}}{M^{1 / 2} Q^{1 / 4}} \\
& +Q^{1 / 2} H^{1 / 2} x^{1-\gamma / 2}+Q^{1 / 4} H^{3 / 4} x^{1-\gamma / 4},
\end{aligned}
$$

while, in the case of $H_{1} \leq H \leq H_{0}$,

$$
\begin{aligned}
S_{\mathrm{II}} \leq & \frac{M^{1 / 2} Q^{1 / 2} H_{1} x^{1 / 2+\varepsilon}}{H^{1 / 2}}+\frac{H_{1} H^{1 / 4} x^{1+\gamma / 4}}{M^{1 / 2} Q^{1 / 4}} \\
& +Q^{1 / 2} H_{1}^{1 / 2} x^{1-\gamma / 2}+\frac{Q^{1 / 4} H_{1} x^{1-\gamma / 4}}{H^{1 / 4}} .
\end{aligned}
$$

In case of (4.4) we choose $Q=x^{1-2 \delta-\varepsilon} / M H$. Then, under the assumptions $x^{b} \leq M \leq x^{c}$, where $b>5(1-\gamma)+6 \delta$ and $c<$ 
$1-(1-\gamma)-2 \delta$, we have $Q \geq 1$, and $S_{\mathrm{II}} \ll x^{1-\delta-\varepsilon}$. Similarly, in case of (4.5) we choose $Q=H x^{1-2 \delta-\varepsilon} / H_{1}^{2} M$ and find that we have $Q \geq 1$ and $S_{\mathrm{II}} \ll x^{1-\delta-\varepsilon}$ under the same assumptions. Summarizing the results of this section we have

Proposition 2. Let $M$ satisfy $x^{b} \leq M \leq x^{c}$ where

$$
b>5(1-\gamma)+6 \delta
$$

and

$$
c<1-(1-\gamma)-2 \delta .
$$

Then, for all sufficiently small positive $\varepsilon$,

$$
S_{\mathrm{II}} \ll x^{1-\delta-\varepsilon} .
$$

5. Type I bilinear forms. We treat (3.4). For any fixed $0 \leq u \leq 1$, $H \leq H_{0}$ we trivially have

$$
\text { (5.1) } \begin{aligned}
\sum_{h \sim H}\left|\sum_{\substack{m n \sim x \\
m \sim M}} a_{m} e\left(\alpha m n+h(m n+u)^{\gamma}\right)\right| \\
\quad \leq \sum_{h \sim H} \sum_{m \sim M}\left|\sum_{n \sim x / m} e\left(\alpha m n+h(m n+u)^{\gamma}\right)\right| .
\end{aligned}
$$

We are going to use two different methods to estimate this. In the first we simply apply Lemma 1 to the innermost sum. Writing $F(n)=$ $\alpha m n+h(m n+u)^{\gamma}$ we can calculate for $n \sim x / m \sim x / M, h \sim H$

$$
F^{\prime \prime}(n)=\gamma(\gamma-1) h m^{2}(m n+u)^{\gamma-2} \asymp x^{\gamma-2} H M^{2} .
$$

Insertion of (2.7) in (5.1) gives that

$$
\begin{aligned}
S_{\mathrm{I}} & \ll \min \left(1, \frac{H_{1}}{H}\right)\left(x^{\gamma / 2} H^{3 / 2} M+x^{1-\gamma / 2} H^{1 / 2}\right) \\
& \ll x^{\gamma / 2} H_{1} H_{0}^{1 / 2} M+x^{1-\gamma / 2} H_{1}^{1 / 2}
\end{aligned}
$$

Thus we have $S_{\mathrm{I}} \ll x^{1-\delta-\varepsilon}$ for all sufficiently small positive $\varepsilon$ provided that

$$
2(1-\gamma)+\delta<1
$$

and $1 \leq M \leq x^{a_{1}}$ where

$$
a_{1}<\frac{1}{2}-(1-\gamma)-\frac{3 \delta}{2} .
$$


Our second method for the estimation of $S_{\mathrm{I}}$ is considerably more complicated. We begin by fixing $h \sim H$ so that

$$
S_{\mathrm{I}} \leq \min \left(1, \frac{H_{1}}{H}\right) \sum_{h \sim H} K_{h}
$$

where

$$
K_{h}=\sum_{m \sim M} \mid \sum_{n \sim x / m} e\left(\alpha m n+h(m n+u)^{\gamma} \mid .\right.
$$

We apply a translation in the variable $n$ together with Cauchy's inequality, a classic technique [Ti, Lemma 5.10] which we use in the form

LEMMA 3 (see Lemma 5 of [HB]). Let I be a sub-interval of $(N, 2 N)$ and let $J$ be a positive integer. Then, for any complex $z_{n}$ we have

$$
\left|\sum_{n \in I} z_{n}\right|^{2} \leq\left(1+N J^{-1}\right) \sum_{j \leq J}\left(1-|j| J^{-1}\right) \sum_{n, n+j \in I} \bar{z}_{n} z_{n+j} .
$$

Applied to $K_{h}$ this yields

$$
\begin{aligned}
K_{h}^{2} & \ll M \sum_{m \sim M}\left|\sum_{n \sim x / m} e\left(\alpha m n+h(m n+u)^{\gamma}\right)\right|^{2} \\
& \ll M(1+x / M J)\left(x+\sum_{j=1}^{J} E_{j}\right)
\end{aligned}
$$

where

$$
E_{j}=\left|\sum_{m \sim M} \sum_{\substack{n \sim x / m \\ n+j \sim x / m}} e\left(\alpha j m+h(m(n+j)+u)^{\gamma}-h(m n+u)^{\gamma}\right)\right|,
$$

and where we have ignored the negative values of $j$, which may be treated in the same manner. Now

$$
\begin{aligned}
& h(m(n+j)+u)^{\gamma}-h(m n+u)^{\gamma} \\
&= h(m(n+j))^{\gamma}-h(m n)^{\gamma} \\
&+\gamma h \int_{0}^{u}\left((m(n+j)+t)^{\gamma-1}-(m n+t)^{\gamma-1}\right) d t \\
&= h(m(n+j))^{\gamma}-h(m n)^{\gamma}+\gamma h \int_{0}^{u} m j(\gamma-1)(\xi(t))^{\gamma-2} d t,
\end{aligned}
$$


for some $\xi \sim x$. Thus

$$
E_{j}=\left|\sum_{m \sim M} \sum_{\substack{n \sim x / m \\ n+j \sim x / m}} e(G(m, n))\right|+O\left(\sum_{m} \sum_{n} h j m x^{\gamma-2}\right)
$$

where $G(m, n)=\alpha j m+h m^{\gamma}\left((n+j)^{\gamma}-n^{\gamma}\right)$ and the second double sum is $\ll H j M x^{\gamma-1}$.

To the first double sum we are going to apply Poisson summation in the variable $n$, followed by an application of van der Corput's method (Lemma 1) to the sum in $m$. In [HB] the same methods were applied but with the variables reversed and with Lemma 1 replaced by a more sophisticated exponent pair. In our case, we need an estimate uniform in $\alpha$ and this forces the change of strategy and the weaker result.

We need the following result which may be proved by replacing $f$ by $-f$ in [HB, Lemma 6$]$.

LEMMA 4. Let $0<a \leq b<2 a$. Let $f(z / a)$ be holomorphic on an open convex set $R$ containing the real line segment $[1, b / a]$ and satisfy $\left|f^{\prime \prime}(z / a)\right| \leq \Delta$ on $R$. let $f(x)$ be real when $x$ is real and let $f^{\prime \prime}(x) \geq c \Delta$ with $c>0$. For every integer $\nu$ with $f^{\prime}(a)<\nu \leq f^{\prime}(b)$ define $n_{\nu}$ by $f^{\prime}\left(n_{\nu}\right)=\nu$. Then

$$
\begin{aligned}
\sum_{a<n \leq b} e(f(n))= & e(-1 / 8) \sum_{\nu}\left(f^{\prime \prime}\left(n_{\nu}\right)\right)^{-1 / 2} e\left(f\left(n_{\nu}\right)-\nu n_{\nu}\right) \\
& +O\left(\Delta^{-1 / 2}\right)+O(\log (2+\Delta(b-a)))
\end{aligned}
$$

where the implied constants depend only on $c$ and $R$.

For given $m$ we take $f(n)=G(m, n)$. We have

$$
\left\{\begin{array}{l}
\partial G / \partial n=\gamma h m^{\gamma}\left((n+j)^{\gamma-1}-n^{\gamma-1}\right) \asymp-h j M^{2} x^{\gamma-2}, \\
\partial^{2} G / \partial n^{2}=\gamma(\gamma-1) h m^{\gamma}\left((n+j)^{\gamma-2}-n^{\gamma-2}\right) \asymp h j M^{3} x^{\gamma-3},
\end{array}\right.
$$

provided that we assume, as we henceforth do, that

$$
J M<x^{1-\varepsilon} .
$$

Applying Lemma 4 to the first double sum in (5.7) we get from the error terms in Lemma 4 a contribution which is bounded by

$$
\sum_{m}\left(\log x+\left(h j M^{3} x^{\gamma-3}\right)^{-1 / 2}\right) \ll x^{\varepsilon}\left(M+(h j M)^{-1 / 2} x^{3 / 2-\gamma / 2}\right) .
$$


For a given integer $\nu \asymp-h j M^{2} x^{\gamma-2}$ we define $n_{\nu}=n_{\nu}(m)$ by

$$
\gamma h m^{\gamma}\left(\left(n_{\nu}+j\right)^{\gamma-1}-n_{\nu}^{\gamma-1}\right)=\nu,
$$

and consider the function

$$
g(m)=G\left(m, n_{\nu}(m)\right)-\nu n_{\nu}(m) .
$$

Combining (5.7), (5.9), (5.10) in Lemma 4, we have

$$
\begin{aligned}
E_{j} \ll & h j M x^{\gamma-1}+M x^{\varepsilon}+(h j M)^{-1 / 2} x^{3 / 2-\gamma / 2+\varepsilon} \\
& +\left|\sum_{\nu} \sum_{m}\left(\frac{\partial^{2} G}{\partial n^{2}}\left(m, n_{\nu}\right)\right)^{-1 / 2} e(g(m))\right|,
\end{aligned}
$$

where, for given $\nu$, one may check that the summation over $m$ determined by the conditions $m \sim M, m n \sim x, m(n+j) \sim x$, $\frac{\partial G}{\partial n}(m, a(m))<\nu \leq \frac{\partial G}{\partial n}(m, b(m))$ is summation over an interval.

We intend to apply Lemma 1 to $\sum_{m}$ and for this purpose we shall need to calculate $g^{\prime \prime}(m)$. We should also like to remove the factor $\left(\frac{\partial^{2} G}{\partial n^{2}}\left(m, n_{\nu}\right)\right)^{-1 / 2}$ by Abel summation, which we may do provided that we show that it is monotonic in $m$. We have

$$
\begin{aligned}
\Delta_{\lambda} & \stackrel{\text { def }}{=}\left(n_{\nu}+j\right)^{\lambda}-n_{\nu}^{\lambda}=\lambda \int_{0}^{j}\left(n_{\nu}+t\right)^{\lambda-1} d t \\
& =\lambda j n_{\nu}^{\lambda-1}+\lambda(\lambda-1) \int_{0}^{j} \int_{0}^{t}\left(n_{\nu}+\tau\right)^{\lambda-2} d \tau d t \\
& =\lambda j n_{\nu}^{\lambda-1}+O\left(j^{2} n_{\nu}^{\lambda-2}\right) .
\end{aligned}
$$

A computation shows that

$$
n_{\nu}^{\prime}(m)=-\frac{\gamma \Delta_{\gamma-1}}{(\gamma-1) m \Delta_{\gamma-2}}
$$

and, using this, we further compute that

$$
\frac{d}{d m}\left(\frac{\partial^{2} G}{\partial n^{2}}\left(m, n_{\nu}\right)\right)=\frac{\gamma^{2} h m^{\gamma-1}}{\Delta_{\gamma-2}}\left\{(\gamma-1) \Delta_{\gamma-2}^{2}-(\gamma-2) \Delta_{\gamma-1} \Delta_{\gamma-3}\right\}
$$

and, by (5.12), this is

$$
=\gamma^{2}(\gamma-1) h j m^{\gamma-1} n^{\gamma-3}\left\{1+O\left(\frac{j}{n}\right)\right\} .
$$

Recalling the assumption $J M<x^{1-\varepsilon}$, this is negative, so that

$$
\left(\frac{\partial^{2} G}{\partial n^{2}}\left(m, n_{\nu}\right)\right)^{-1 / 2} \text { is monotonic increasing in } m \text {. }
$$


Using (5.13) we also compute

$$
g^{\prime}(m)=\alpha j+\gamma h m^{\gamma-1} \Delta_{\gamma}
$$

and

$$
\begin{aligned}
g^{\prime \prime}(m) & =\gamma(\gamma-1) h m^{\gamma-2} \Delta_{\gamma}+\gamma^{2} h m^{\gamma-1} \Delta_{\gamma-1} n_{\nu}^{\prime}(m) \\
& =\frac{\gamma h\left((\gamma-1)^{2} \Delta_{\gamma} \Delta_{\gamma-2}-\gamma^{2} \Delta_{\gamma-1}^{2}\right)}{(\gamma-1) m^{2-\gamma} \Delta_{\gamma-2}} .
\end{aligned}
$$

By (5.12) we have

$$
g^{\prime \prime}(m)=-\frac{2 \gamma^{2}(\gamma-1)}{\gamma-2} h j n_{\nu}^{\gamma-1} m^{\gamma-2}\left(1+O\left(\frac{j}{n_{\nu}}\right)\right)
$$

and since $J M<x^{1-\varepsilon}$, we have

$$
g^{\prime \prime}(m) \asymp h j M^{-1} x^{\gamma-1} .
$$

We use (5.8), (5.14), (5.15) to apply Abel summation and then Lemma 1 to the sum in (5.11). These yield

$$
\begin{aligned}
\sum_{\nu} \sum_{m} & \ll \frac{h j M^{2} x^{\gamma-2}}{\left(h j M^{3} x^{\gamma-3}\right)^{1 / 2}}\left(M h^{1 / 2} j^{1 / 2} M^{-1 / 2} x^{\gamma / 2-1 / 2}\right. \\
& \left.+h^{-1 / 2} j^{-1 / 2} M^{1 / 2} x^{1 / 2-\gamma / 2}\right) \\
\ll & h j M x^{\gamma-1}+M .
\end{aligned}
$$

From (5.6) and (5.11) this gives, for $J M<x^{1-\varepsilon}$,

$$
K_{h}^{2} \ll J^{-1} x^{1+\varepsilon}\left(x+h J^{2} M x^{\gamma-1}+h^{-1 / 2} J^{1 / 2} M^{-1 / 2} x^{3 / 2-\gamma / 2}\right)
$$

and so, by (5.4),

$$
\begin{array}{r}
S_{\mathrm{I}} \ll x^{\varepsilon}\left(\min \left(1, \frac{H_{1}}{H}\right)\right)\left(H J^{-1 / 2} x+H^{3 / 2} J^{1 / 2} M^{1 / 2} x^{\gamma / 2}\right. \\
\left.+H^{3 / 4} J^{-1 / 4} M^{-1 / 4} x^{5 / 4-\gamma / 4}\right) .
\end{array}
$$

We choose $J=\left[H^{-1 / 2} M^{-1 / 2} x^{1-\gamma / 2}\right]$ and note that $1 \leq J<$ $M^{-1} x^{1-\varepsilon}$ as required, provided that $M<x^{1-\delta}, M<x^{1-(1-\gamma)-\varepsilon}$. With this choice of $J$, we have

$$
\begin{aligned}
S_{1} & \ll x^{\varepsilon}\left(\min \left(1, \frac{H_{1}}{H}\right)\right)\left(H^{5 / 4} M^{1 / 4} x^{1 / 2+\gamma / 4}+H^{7 / 8} M^{-1 / 8} x^{1-\gamma / 8}\right)^{?} \\
& \ll x^{\varepsilon}\left(H_{1} H_{0}^{1 / 4} M^{1 / 4} x^{1 / 2+\gamma / 4}+H_{1}^{7 / 8} M^{-1 / 8} x^{1-\gamma / 8}\right) \\
& \ll x^{\varepsilon}\left(M^{1 / 4} x^{7 / 4-\gamma+\delta / 4}+M^{-1 / 8} x^{15 / 8-\gamma}\right)
\end{aligned}
$$


and this is $\ll x^{1-\delta-\varepsilon}$ provided that $x^{a_{2}}<M \leq x^{a}$ where

$$
a_{2}>-1+8(1-\gamma)+8 \delta
$$

and

$$
a<1-4(1-\gamma)-5 \delta .
$$

Comparing (5.3) and (5.16) we see that, under the condition

$$
6(1-\gamma)+(19 / 3) \delta<1,
$$

the upper bound for $a_{1}$ exceeds the lower bound for $a_{2}$ and the two methods overlap. Summarizing the results of this section we have

Proposition 3. Assume that (5.18) holds. Let $M$ satisfy $1 \leq M \leq$ $x^{a}$ where a satisfies (5.17). Then, for all sufficiently small positive $\varepsilon$,

$$
S_{\mathrm{I}} \ll x^{1-\delta-\varepsilon} .
$$

6. Conclusion. In this section we combine Propositions $1,2,3$ to complete the proof of (2.10), hence of Theorem 4, and hence of Theorem 1. By these three propositions it remains only to show that the constants

$$
\begin{aligned}
& a=1-4(1-\gamma)-5 \delta-\varepsilon, \\
& b=5(1-\gamma)+6 \delta+\varepsilon, \\
& c=1-(1-\gamma)-2 \delta-\varepsilon,
\end{aligned}
$$

which obviously satisfy (5.17), (4.6), and (4.7), also satisfy, provided $\varepsilon$ is sufficiently small, the conditions (3.7), (3.8), (3.9).

The condition (3.7), $b<2 / 3$ follows for all sufficiently small $\varepsilon$ since $(15 / 2)(1-\gamma)+9 \delta<1$. The condition (3.8), $1-c<c-b$ similarly follows since $7(1-\gamma)+10 \delta<1$. The condition (3.9), $1-a<c / 2$, which is the most difficult to satisfy, follows since $9(1-\gamma)+12 \delta<1$. This completes the proof.

\section{REFERENCES}

[De] J.-M. Deshouillers, Nombres premiers de la forme [ $\left.n^{c}\right]$, C. R. Acad. Sci. Paris, Ser. A-B, 282 (3) (1976), A131-A133.

[EN] P. Erdős and M. B. Nathanson, Lagrange's Theorem and Thin Subsequences of Squares, Contributions to Probability (J. Gani, V. K. Rohatgi, eds.), Academic Press, New York, 1981, 3-9.

[HB] D. R. Heath-Brown, The Pjateckii-Šapiro prime number theorem, J. Number Theory, 16 (1983) 242-266. 
[HB2] __ Prime numbers in short intervals and a generalized Vaughan identity, Canad. J. Math., 34 (1982), 1365-1377.

[K] G. Kolesnik, Primes of the form [ $\left.n^{c}\right]$, Pacific J. Math., 118 (1985), 437-447.

[PS] I. I. Piatetski-Shapiro, On the distribution of prime numbers in sequences of the form $[f(m)]$, Mat. Sb., 33 (1953), 559-566.

[Ti] E. C. Titchmarsh, The Theory of the Riemann Zeta-function, Oxford, London, 1951.

[Va] R. C. Vaughan, The Hardy-Littlewood Method, Cambridge Tracts in Math. 80, Cambridge University Press, Cambridge, 1981.

[Vi] I. M. Vinogradov, Representation of an odd number as the sum of three primes, Dokl. Akad. Nauk SSSR, 15 (1937), 291-294.

[Wi] E. Wirsing, Thin subbases, Analysis, 6 (1986), 285-308.

Received April 14, 1991. Research of both authors partially supported by NSERC Grant \#A5123.

SCARborough COLlege

UNIVERSITY OF TORONTO

SCARBorough, Ontario MIC 1A4 CANADA

AND

Mathematics INSTITUTE

HungaRian ACADEMY OF SCIENCES

BUDAPEST, HUNGARY 


\title{
PACIFIC JOURNAL OF MATHEMATICS
}

Founded by

\author{
E. F. BECKENBACH (1906-1982) F. WolF (1904-1989) \\ EDITORS
}

\author{
V. S. VARADARAJAN \\ (Managing Editor) \\ University of California \\ Los Angeles, CA 90024-1555 \\ vsv@math.ucla.edu \\ Herbert Clemens \\ University of Utah \\ Salt Lake City, UT 84112 \\ clemens@math.utah.edu \\ F. Michael Christ \\ University of California \\ Los Angeles, CA 90024-1555 \\ christ@math.ucla.edu \\ ThOMAs ENRIGHT \\ University of California, San Diego \\ La Jolla, CA 92093 \\ tenright@ucsd.edu
}

\author{
Nicholas ERCOLANI \\ University of Arizona \\ Tucson, AZ 85721 \\ ercolani@math.arizona.edu \\ R. FINN \\ Stanford University \\ Stanford, CA 94305 \\ finn@gauss.stanford.edu \\ VAUGHAN F. R. JONES \\ University of California \\ Berkeley, CA 94720 \\ vfr@math.berkeley.edu \\ Steven KerckhofF \\ Stanford University \\ Stanford, CA 94305 \\ spk@gauss.stanford.edu
}

C. C. Moore

University of California

Berkeley, CA 94720

MaRTin SCHARLEMANN

University of California

Santa Barbara, CA 93106

mgscharl@henri.ucsb.edu

HAROLD STARK

University of California, San Diego

La Jolla, CA 92093

\author{
UNIVERSITY OF ARIZONA \\ UNIVERSITY OF BRITISH COLUMBIA \\ CALIFORNIA INSTITUTE OF TECHNOLOGY \\ UNIVERSITY OF CALIFORNIA \\ MONTANA STATE UNIVERSITY \\ UNIVERSITY OF NEVADA, RENO \\ NEW MEXICO STATE UNIVERSITY \\ OREGON STATE UNIVERSITY
}

\section{SUPPORTING INSTITUTIONS}

UNIVERSITY OF OREGON

UNIVERSITY OF SOUTHERN CALIFORNIA

STANFORD UNIVERSITY

UNIVERSITY OF HAWAII

UNIVERSITY OF TOKYO

UNIVERSITY OF UTAH

WASHINGTON STATE UNIVERSITY

UNIVERSITY OF WASHINGTON 


\section{PACIFIC JOURNAL OF MATHEMATICS}

Volume $156 \quad$ No. $1 \quad$ November 1992

Enveloping algebras of Lie groups with discrete series

Nguyen HuU AnH and VuOng Manh Son

Asymptotic behavior of eigenvalues for a class of pseudodifferential operators on $\mathbf{R}^{n}$

JUNICHI ARAMAKI

A hybrid of theorems of Vinogradov and Piatetski-Shapiro

ANTAL BALOG and JOHN BENJAMIN FRIEDLANDER

Chaos in terms of the map $x \rightarrow \omega(x, f)$

ANDREW MiCHAEL BRUCKNER and JACK GARY CEDER

Local real analytic boundary regularity of an integral solution operator 97 of the $\bar{\partial}$-equation on convex domains

ZHENHUA CHEN

On some properties of exhaustion maps between bounded domains

Chi Keung Cheung

A generalization of maximal functions on compact semisimple Lie groups

HENDRA GUNAWAN

Stability of nonsingular group orbits

CLARK DEAN HORTON

Bordism and regular homotopy of low-dimensional immersions

JOHN FORBES HuGHES

On six-connected finite $H$-spaces

JAMES PEICHENG LiN and Frank WiLliams 